\title{
Is There Any Association Between Childhood Traumatic Experiences, Dissociation and Psychotic Symptoms in Schziophrenic Patients?
}

\author{
Zeynep Yildiz Akbey ${ }^{1}$, Mustafa Yildiz², and Nermin Gündüz ${ }^{1 凶}$ \\ ${ }^{1}$ Department of Psychiatry, Faculty of Medicine, Kutahya Health Sciences University, Kutahya, Turkey \\ ${ }^{2}$ Department of Psychiatry, Faculty of Medicine, Kocaeli University, Kocaeli, Turkey
}

Objective The aim of this study was to investigate the rates childhood traumatic experiences (CTEs) of schizophrenia patients and to examine relationship between childhood traumatic experiences, dissociation and psychotic symptoms.

Methods One hundred schizophrenia patients who had 5 or 5 points below in Clinical Global Impression-Severity (CGI-S) and who were not in active psychotic episode and 50 healthy siblings who grew up in the same environment with the patients were included to the study. Structured Clinical Interview for DSM IV, Scale for the Assessment of Positive Symptoms, Scale for the Assessment of Negative Symptoms, Dissociative Experiences Scale (DES), CGI-S, Global Assessment of Functioning Scale and Childhood Trauma Questionnaire (CTQ-28) applied to the patients.

Results Childhood abuse subscales and DES scores were statistically higher in the patient group ( $<<0.001)$. We determined moderately significant positive correlation between CTQ-28 and DES. We determined moderately significant positive correlation between CTQ total scores and persecutory delusions, delusions of reference, ability to feel intimacy and closeness; relationship with friends and peers. Also, there was a significant positive correlation between persecutory delusions and CTQ-total, DES-total and all subscales of CTQ-28.

Conclusion Clinicians should inquire about CTEs to develop comprehensive formulations and treatment plans among schizophrenia.

Psychiatry Investig 2019;16(5):346-354

Key Words Schizophrenia, Childhood trauma, Dissociation.

\section{INTRODUCTION}

Genetic susceptibility to schizophrenia may be considerably determinative in pathogenesis. ${ }^{1}$ However, the fact that the risk of schizophrenia is not $100 \%$ in genetic concordance twins suggests that different etiologic and pathophysiologic mechanisms, including environmental factors, play a significant role in schizophrenia. ${ }^{1,2}$ In this respect, some researchers take into account childhood traumatic experiences (CTEs) (sexual abuse, physical abuse, emotional/psychological abuse and neglect) as a possible potential environmental risk factor that may increase the vulnerability to schizophrenia.,4

History of CTEs, including all specific forms of traumas,

Received: December 26, 2018 Accepted: February 10, 2019

$\triangle$ Correspondence: Nermin Gündüz, MD

Department of Psychiatry, Faculty of Medicine, Kutahya Health Sciences University, Kütahya Sağlık Bilimleri Üniversitesi Evliya Çelebi Yerleşkesi, Tavşanlı Yolu, 10. Km Kutahya, Turkey

Tel: +90-274 26000 43-44-45-46, Fax: +90-2742652285

E-mail: ngunduz2798@hotmail.com

(a) This is an Open Access article distributed under the terms of the Creative Commons Attribution Non-Commercial License (https://creativecommons.org/licenses/bync/4.0) which permits unrestricted non-commercial use, distribution, and reproduction in any medium, provided the original work is properly cited. has been extensively investigated as substantially increasing the risk of psychosis with the odds ratios (ORs) of between 2.78 and $3.60 .^{5,6}$ In addition, in a meta-analysis study evaluating the prevalence of self-reported childhood sexual (CSA), physical (CPA) and emotional abuse (CEA) in psychotic patients, the prevalence of self-reported CSA, CPA, CEA were respectively found to be $26 \%, 39 \%$ and $34 \%$. $^{7}$ This findings were replicated in other studies. ${ }^{8-10}$

It is also essential by which mechanisms CTEs may increase the risk for psychosis. Relevant studies have shown that CTEs such as CPA and CSA can increase the risk of early onset psychotic symptoms, ${ }^{11}$ psychosis-like clinical symptoms, ${ }^{12}$ schizophrenia, and other psychotic disorders ${ }^{13}$ leading to psychological and biological changes in individuals. Some researchers have revealed a causal relationship between CTEs and schizophrenia in some schizophrenia patients according to the Traumagenic Neurodevelopmental Model. This model suggests the hypersensitivity to stress commonly described in psychotic patients and it may be the result of neurodevelopmental alterations in the structures of the brain following early life traumatic experiences. ${ }^{14,15}$ 
The relation between CTEs and positive and negative psychotic symptoms was also studied. Some researchers have speculated that specific type of CTEs may facilitate the specific symptoms in adult schizophrenia patients. ${ }^{16,17}$ In general, while CTEs are associated with positive psychotic symptoms, ${ }^{12,16-18}$ findings about negative symptoms appear to be more complex. ${ }^{16,17}$ In a study conducted on first episode psychotic patients, history of sexual assault was associated with both positive and negative symptoms. ${ }^{18}$ In a 2 -year follow-up prospective population-based study, it was found that early CTEs increase the risk for positive psychotic symptoms. ${ }^{12}$ Also, in a study conducted by Gallagher and Jones ${ }^{16}$ childhood neglect was found to be associated with negative symptoms of schizophrenia and childhood abuse was related with positive symptoms of the psychosis.

The relation between specific types of CTEs and psychotic symptoms is important too. For example, while childhood abuse was often found to be associated with positive symptoms, childhood neglect was often found to be associated with negative symptoms. ${ }^{16}$ Researchers have also focused on the link between the development of specific symptoms of psychosis such as auditory hallucinations ${ }^{9,19,20}$ or delusions or,21,22 $^{9}$ and CTEs specific types such as CPA, CEA or CSA., ${ }^{9,1321}$ As a result, it seems more likely that CTEs are related to a particular symptom group, rather than categorical diagnosis like schizophrenia. ${ }^{23}$ Notwithstanding the above, there is no linear or straightforward relationship between CTEs and schizophrenia. Also, CTEs is neither necessary nor sufficient alone for the risk of schizophrenia. ${ }^{24}$

There are also studies investigating the relationship between CTEs, dissociation and psychosis. ${ }^{10,25,26}$ Also, dissociation may coincide with positive psychotic symptoms, especially in the acute psychotic episode. Thus, there is an important assosiation between dissociative symptoms, CTEs and psychotic symptoms. ${ }^{27}$

Much of the evidence for the association between schizophrenia and CTEs has been obtained from studies conducted in Western societies, whereas few studies were conducted on Eastern culture. ${ }^{9,10,28}$ Also, there are very few studies investigating the relation between childhood specific traumatic forms and schizophrenia symptoms or symptom clusters in Eastern culture until now. ${ }^{9,28,29}$

Thus, first, the aim of this study wass to investigate whether there is a difference in the rates of CTEs of schizophrenia patients and their healthy siblings living in the same environment in a sample of Eastern culture. The second aim was to examine relationships between a broad range of childhood specific adversities (including the abuse and neglect) and the presence of DSM-IV characteristic symptoms of schizophrenia: hallucinations, delusions, negative symptoms. Finally, we wanted to know if there is relationship between dissociation, CTEs and psychotic symptoms.

\section{METHODS}

\section{Study participants}

The patients followed up with the diagnosis of schizophrenia by Kocaeli University Faculty of Medicine outpatient clinics, and their healthy siblings who grew up in the same environment with the patients were enrolled in the study. 100 schizophrenia patients who had 5 or fewer points in Clinical Global Impression-Severity (CGI-S) and who were not having an active psychotic episode and 50 healthy siblings who grew up in the same environment with the patients like the patient group in terms of age, gender and education as a control group were included in the study. The study was approved by the Kocaeli University Ethics Committee for Clinical Trials with the number of KOÜ KAEK 2012/55. A written informed consent was obtained from each participant. The study was conducted in accordance with the principles of the Declaration of Helsinki.

Study inclusion criteria for patients were determined as to have a diagnosis of schizophrenia according to DSM-IV, ${ }^{30}$ to be in the age range of $18^{-60}$, to be at least literate, to have no language problem in terms of communication, not to have Electroconvulsive Therapy (ECT) in the last 3 months.

Study inclusion criteria for the control group was determined to grow up in the same environment with the schizophrenia sibling and to be similar to the patient group regarding age, gender and education. Patients who are illiterate, who have significant visual, hearing and cognitive impairment of communication, patients with mental retardation, those with substance and alcohol use disorder, those with the chronic neurological disease with significant cognitive decline such as epilepsy, Parkinson were excluded. Exclusion criteria were the same for the control group.

A sociodemographic data form, Structured Clinical Interview for DSM-IV axis I disorders (SCID-I), Scale for the assessment of positive symptoms (SAPS), scale for the assessment of negative symptoms (SANS), dissociative experiences scale (DES), CGI-S, Global Assessment of Functioning Scale (GAF), and Childhood Trauma Questionnaire (CTQ-28) were applied to the patients. Sociodemographic data form, DES and CTQ-28 were applied to the control group.

\section{Instruments}

\section{Sociodemographic data form}

This form was prepared to evaluate demographic characteristics of the patients like age, gender, education year, marital status, type of schizophrenia, the age of illness onset, du- 
ration of illness, number and duration of hospitalization and psychiatric comorbidity.

\section{Dissociative experiences scale}

It is a scale of 28 items developed by Bernstein and Putnam. Scores above 30 on the scale indicate the presence of dissociative disorder. ${ }^{31}$ Turkish validity and reliability study was conducted by Yargıç et al..$^{32}$

\section{Childhood trauma questionnaire}

This 28-item retrospective questionnaire investigates the presence of the traumatic experiences like abuse and neglect, during childhood and adolescence. It was developed by Bernstein and colleagues. This self-reported form consists of 5 sub-dimensions of emotional neglect, physical neglect, sexual abuse, emotional abuse and physical abuse. ${ }^{33}$ It was first developed as 70 items and reduced to 53 items in the following years. Şar et al. ${ }^{34}$ shortened that form and adapted to Turkish as CTQ28. 28 question form was used in this study.

\section{Global assessment of functioning scale}

It is used to assess the mental, social and occupational functioning of the patient on axis V of the DSM-IV. The clinician examining the patient assesses the overall level of adjustment of the patient over a score ranging from $0-100$ using his or her judgment. ${ }^{30}$

\section{Scale for the assessment of positive symptoms}

It was developed by Andreasen ${ }^{35}$ Turkish validity and reliability study was conducted by Erkoç et al. ${ }^{36}$

\section{Scale for the assessment of negative symptoms}

It was developed by Andreasen ${ }^{35}$ Turkish validity and reliability study was conducted by Erkoç et al. ${ }^{37}$

\section{Structured clinical interview for DSM-IV}

This is a structured clinical interview developed for use by mental health professionals. SCID-I allows diagnosing both present and lifetime Axis I mental disorders. ${ }^{38}$ For the reliability of the Turkish form, the overall percent agreement for all diagnoses was $98.1 \%$, and the kappa coefficient was $0.86 .{ }^{39}$

\section{Clinical global impression-severity}

This scale is used for general evaluation of mental disorders. ${ }^{40}$ Clinical impression is scored between 1 (undetected) and 7 (the most severe patients). High scores on the scale indicate that the clinical symptoms are severe.

\section{Statistical analysis}

Data were analyzed using the Statistical Program for Social
Sciences (SPSS version 13; SPSS Inc., Chicago, IL, USA). The appropriate parametric and nonparametric tests were used for testing whether the data has a statistically normal distribution property. To compare the differences between the groups, the chi-square test was used for categorical variables, and the Mann-Whitney U test was used for mean values of continuous variables. Pearson Correlation Tests were used to examine the relationship between CTQ, DES, SAPS, SANS, the age of illness onset, CGI-S and GAF. Results were evaluated by taking $\mathrm{p}<0.05$ level of significance into consideration. Correlation strength is weak if $\mathrm{r}=0.00-0.24$, moderate if $\mathrm{r}=0.25-0.49$, strong if $\mathrm{r}=0.50-0.74$, and very strong if $\mathrm{r}=0.75-1.00 .{ }^{41}$

\section{RESULTS}

\section{Sociodemographic characteristics}

100 patients diagnosed with schizophrenia and 50 healthy siblings were included in this study. There was a significant difference regarding marital status while there was no difference between the groups regarding age, gender and education year. The mean age of the groups were $38.7 \pm 10.5$ years for patients and $35.3 \pm 10.4$ years for healthy controls $(\mathrm{p}=0.059)$. The mean of education year of the groups $8.5 \pm 3.4$ for patients and $8.8 \pm 3.4$ for healthy controls $(\mathrm{p}=0.669)$.

In the patient group mean age of illness onset was $24.4 \pm 8.9$; mean duration of illness was $14.1 \pm 8.5$ years; a mean number of hospitalizations was $3.0 \pm 2.7$; mean duration of hospitalization was $78.4 \pm 70.4$ days. Sociodemographic characteristics of the patient and the control group are presented in Table 1.

Although there were no statistically significant differences between groups in terms CTQ-28 total and neglect (CPN and CEA) subscales, there was statistically significant differences between groups in terms abuse (CPA, CSA, CEA) subscales. There were also statistically significant differences between groups in terms DES total scores $(\mathrm{p}<0.001)$. Mean SAPS score was $36.18 \pm 21.53$, mean SANS score was $47.01 \pm 21.81$; mean CGI-S score was $3.02 \pm 1.27$, and the mean score of GAF was $63.35 \pm 10.00$ in the patient group. Mean scores of the scales are presented in detail in Table 2.

Correlations between CTQ-28 total and CTQ-28 subscales and DES total scores are presented at Table 3. There was a moderately significant positive correlation between CTQ-28 total and CTQ-28 subscales and DES total scores.

Correlations between CTQ-28 and DES with age at illness onset, CGI-S, GAF are presented in Table 3. There was a moderately significant positive correlation between DES total scores and CGI-S. There was also a significant positive correlation between GAF and CTQ-total scores and CGI-S (Table 4).

Correlations between CTQ-28, CTQ subscales and DES with SAPS and some psychotic symptoms are presented in 
Table 5.

Correlations between CTQ-28 and DES with some negative symptoms are presented in Table 5.

There was a moderately significant positive correlation between CTQ total scores and persecutory delusions, delusions of reference, ability to feel intimacy and closeness; relationship with friends and peers.

There was a moderately significant positive correlation between DES scores and persecutory delusions, delusions of reference, physical anergia, recreational interests and activities, the ability to feel intimacy and closeness; relationship with friends and peers.

Also, there was a significant positive correlation between persecutory delusions and CTQ-total, DES-total and all subscales of CTQ-28. There was a significant positive correlation

Table 1. Sociodemographic characteristics of the patient and control groups

\begin{tabular}{lccc}
\hline & $\begin{array}{c}\text { Patient } \\
(\mathrm{N}, \%)\end{array}$ & $\begin{array}{c}\text { Control } \\
(\mathrm{N}, \%)\end{array}$ & p value \\
\hline Gender & $69(69)$ & $28(56)$ & $0.116^{*}$ \\
Male & & & $0.020^{*}$ \\
Marital status & $31(31)$ & $27(54)$ & \\
Married & $69(69)$ & $23(46)$ & \\
Single or divorced & & & \\
Type of schziophrenia & $60(60)$ & & \\
Paranoid & $25(25)$ & & \\
Undifferentiated & $13(13)$ & & \\
Residual & $2(2)$ & & \\
Desorganised & & & \\
Comorbide psychiatric diagnosis & & & \\
Mood disorder & $27(27)$ & & \\
SUD & $10(10)$ & & \\
Anxiety disorder & $8(8)$ & & \\
PTSD & $4(4)$ & & \\
APD & $1(1)$ & & \\
Education & $8.5 \pm 3.4$ & $8.8 \pm 3.4$ & \\
Years & & & \\
\hline
\end{tabular}

${ }^{*}$ chi-square test, ${ }^{* *}$ t test. SUD: substance use disorder, PTSD: posttraumatic stress disorder, APD: antisocial personality disorder between Delusions of reference and CTQ-total, DES-total and all subscales of CTQ-28 (Table 5).

\section{DISCUSSION}

Although the patient group reported higher rates of CPA, CSA and CEA, consistent with some studies, ${ }^{7-9,13,28,42}$ they did not report higher rates of childhood trauma compared with healthy siblings in contrast to some studies. ${ }^{24,43}$ Similar with our results, recently in a study conducted by Alameda et al. ${ }^{44}$ researchers found that $24.8 \%$ of the patient group had a documented history of CSA and/or CPA in early psychosis patients. Supporting our findings about CPA, Fisher et al. ${ }^{42}$ found that schizophrenia is associated particularly with CPA in their large population-based case-control study of childhood abuse and psychosis. In a 30-year follow up period, a prospective case-control study conducted to determine whether CSA is a risk factor for later life psychosis, rates of psychotic disorders including schizophrenia, were found to be higher among individuals with a history of CSA compared with those not having a history of CSA controls. ${ }^{13}$

Table 2. Mean scores of the scales

\begin{tabular}{lccccc}
\hline \multirow{2}{*}{ Scales } & Patient & & Control & \\
\cline { 2 - 2 } & Mean \pm SD & & Mean \pm SD & \\
\hline CTQ total & $45.17 \pm 16.38$ & & $40.08 \pm 9.50$ & $\mathrm{p}=0.210$ \\
Physical abuse & $7.57 \pm 3.68$ & & $5.72 \pm 1.61$ & $\mathrm{p}<0.001^{*}$ \\
Sexual abuse & $6.61 \pm 3.43$ & & $5.30 \pm 0.76$ & $\mathrm{p}=0.009^{*}$ \\
Emotional abuse & $9.08 \pm 4.63$ & & $7.36 \pm 2.70$ & $\mathrm{p}=0.033^{*}$ \\
Physical neglect & $9.42 \pm 3.61$ & & $8.58 \pm 2.80$ & $\mathrm{p}=0.276$ \\
Emotional neglect & $12.57 \pm 5.18$ & & $13.12 \pm 5.17$ & $\mathrm{p}=0.510$ \\
DES & $22.62 \pm 17.62$ & & $10.80 \pm 11.76$ & $\mathrm{p}<0.001^{*}$ \\
GAF & $63.35 \pm 10.00$ & & \\
CGI-S & $3.02 \pm 1.27$ & & \\
SAPS & $36.18 \pm 21.53$ & & \\
SANS & $47.01 \pm 21.81$ & &
\end{tabular}

*Mann Whitney U test. CTQ total: Childhood Trauma Questionnaire, DES: Dissociative Experiences Scale, GAF: Global Assessment of Functioning Scale, CGI-S: Clinical Global Impression-Severity, SAPS: Scale for the assessment of positive symptoms, SANS: Scale for the assessment of negative symptoms

Table 3. Correlation between CTQ-28 and DES with age of illness onset, CGI-S, GAS

\begin{tabular}{lccccccc}
\hline & CPA & CEA & CSA & CPN & CEN & CTQ-total & DES-total \\
\hline Onset of illness & $-0.289^{* *}$ & $-0.319^{* *}$ & $-0.233^{*}$ & $-0.408^{* *}$ & $-0.324^{* *}$ & $-0.396^{* *}$ & $-0.188^{*}$ \\
GAF & 0.054 & 0.147 & 0.192 & 0.196 & $0.257^{*}$ & $0.219^{*}$ & $0.242^{*}$ \\
CGI-S & -0.030 & -0.149 & $-0.222^{*}$ & -0.165 & -0.196 & $-0.197^{*}$ & $-0.264^{* *}$ \\
\hline
\end{tabular}

Pearson Correlation Test. ${ }^{*} \mathrm{p}<0.05,{ }^{* *} \mathrm{p}<0.01$. CPA: Childhood Physical Abuse, CEA: Childhood Emotional Abuse, CSA: Childhood Sexual Abuse, CPN: Childhood Physical Neglect, CEN: Childhood Emotional Neglect, CTQ: Childhood Trauma Questionnaire, DES: Dissociative Experiences Scale 
Table 4. Correlation between CTQ-28, CTQ subscales and DES with SAPS some positive psychotic symptoms

\begin{tabular}{llllllcc}
\hline & CPA & CEA & CSA & CPN & CEN & CTQ-total & DES-total \\
\hline SAPS total & 0.057 & 0.071 & 0.183 & 0.027 & 0.037 & 0.089 & 0.192 \\
Auditory hallucinations & 0.069 & 0.028 & $0.206^{*}$ & 0.049 & 0.029 & 0.085 & 0.250 \\
Somatic hallucinations & 0.075 & 0.054 & $0.305^{* *}$ & -0.102 & -0.014 & 0.068 & 0.131 \\
Persecutory delusions & $0.240^{*}$ & $0.298^{* *}$ & $0.293^{* *}$ & $0.270^{* *}$ & $0.228^{*}$ & $0.336^{* *}$ & $0.274^{* *}$ \\
Somatic delusions & 0.185 & $0.220^{*}$ & $0.384^{* *}$ & -0.035 & 0.028 & 0.185 & 0.152 \\
Delusions of reference & $0.218^{*}$ & $0.283^{* *}$ & $0.249^{*}$ & $0.269^{* *}$ & $0.212^{*}$ & $0.312^{* *}$ & $0.278^{* *}$ \\
\hline
\end{tabular}

Pearson Correlation Tests. ${ }^{*} \mathrm{p}<0.05,{ }^{* *} \mathrm{p}<0.01$. SAPS: Scale for the Assessment of Positive Symptoms, CPA: Childhood Physical Abuse, CEA: Childhood Emotional Abuse, CSA: Childhood Sexual Abuse, CPN: Childhood Physical Neglect, CEN : Childhood Emotional Neglect, CTQ: Childhood Trauma Questionnaire, DES: Dissociative Experiences Scale

Table 5. Correlation between CTQ-28 and DES with some negative symptoms

\begin{tabular}{llllllcc}
\hline & CPA & CEA & CSA & CPN & CEN & CTQ-total & DES-total \\
\hline Affective nonresponsivity & 0.008 & 0.102 & 0.098 & 0.104 & $0.238^{*}$ & 0.147 & 0.127 \\
Physical anergia & 0.036 & 0.156 & 0.113 & $0.275^{* *}$ & $0.243^{*}$ & $0.208^{*}$ & $0.260^{* *}$ \\
Recreational interests and activities & -0.009 & 0.143 & 0.055 & $0.239^{*}$ & 0.196 & 0.162 & $0.278^{* *}$ \\
Ability to feel intimacy and closeness & 0.084 & $0.204^{*}$ & 0.147 & $0.280^{* *}$ & $0.333^{* *}$ & $0.272^{* *}$ & $0.306^{* *}$ \\
Relationship with friends and peers & 0.119 & $0.249^{*}$ & 0.194 & $0.320^{* *}$ & $0.292^{* *}$ & $0.298^{* *}$ & $0.280^{* *}$ \\
SANS total & -0.056 & 0.084 & 0.064 & $0.230^{*}$ & $0.219^{*}$ & 0.153 & $0.222^{*}$ \\
\hline
\end{tabular}

Pearson Correlation Tests. ${ }^{*} \mathrm{p}<0.05,{ }^{* *} \mathrm{p}<0.01 . \mathrm{CPA}$ : Childhood Physical Abuse, CEA: Childhood Emotional Abuse, CSA: Childhood Sexual Abuse, CPN: Childhood Physical Neglect, CEN: Childhood Emotional Neglect, CTQ: Childhood Trauma Questionnaire, DES: Dissociative Experiences Scale

These differences in the prevalence of CTEs may be due to some reasons. First, it may be because of the properties of patients. Our study group was not a homogeneous group like consisting only early onset psychotic symptoms, psychosislike clinical symptoms, early psychosis patients or chronic psychosis patients. Second, the sociocultural characteristics of the population in which the research is conducted are also important. ${ }^{45}$ Much of the evidence for the relationship between schizophrenia and CTEs has been obtained from studies conducted in Western societies. The influence of CTEs in Eastern culture, where parenting relationships and social structure differ significantly from Western culture, may be different. ${ }^{9,28,29}$ Finally, different methods used for collecting data from patients such as interviewing with patients, using retrospective data, different measures or scales can affect the results of the studies. ${ }^{2}$ We analyzed CTEs using a self-report questionnaire based on a retrospective assessment of CTEs. Most of the studies evaluating the associations between psychosis and CTEs relied on retrospective assessments. This is often because of expense, extremely long prospective follow-up period of the patients. Also, there are some ethical problems related to prospective longitudinal examinations. ${ }^{2,46}$ Relevant literature suggests the evidence that retrospective analysis of CTEs tends to underestimate rather than exaggerate real incidence rates. ${ }^{46}$ The result of our study can be because of underestimating the rates of CTEs too. Studies have also demonstrated the validity and reliability of trauma-related retrospective reports in psychotic samples, indicating that 1) early life traumatic experiences are stable over a long period, 2) unaffected by current psychopathology, and 3) similar when obtained by other sources of information such as different assessment instruments. ${ }^{47}$ Also it can be possible that schizophrenia patients with having active psychotic episode accompanied by disinhibition may have a greater tendency to display the exact nature of the abuse. ${ }^{47}$ Our study group included schizophrenia patients who had 5 or fewer points in CGI-S and who were not having active psychotic episode. These characteristics of our patient group may contribute to the findings of our study. Although recent meta-analysis comparing the rates of CTEs across different countries revealed that Lower and Middle-Income Countries (LMIC) had higher rates than upper-income countries (UIC), ${ }^{45}$ our findings are not consistent with the results of this meta-analysis. In particular, it was well established that LMICs have higher rates of crime, poverty, and unemployment-all of which are socio-economic factors known to affect the rates of CTEs. ${ }^{45}$ Since we have conducted our study in tertiary care in a big city center, our results are found to be similar to the studies conducted on UIC.

In the second part of the study, we have hypothesized that specific types of CTEs appear to have different effects on particular cognitive processes and at least seem to differ in the psychotic symptoms they are related. In regard to positive psy- 
chotic symptoms, particularly hallucinations, we have tested the hypotheses that severe childhood trauma, particularly CSA, would likely disrupt the source monitoring processes necessary for discriminating between thoughts and external stimuli. We found significant positive relation between CSA and auditory and somatic hallucinations consistent with some studies in the literature. ${ }^{17,20,21,48,49}$ Thus the hypothesis (e.g., ${ }^{49,50}$ ) that differential associations exist between particular adversities and psychotic symptoms was supported by our results.

One of the possible explanation for this significant association between CTEs and schizophrenia as well as the association between CSA and auditory hallucinations, is the diathesis-stress model of schizophrenia. ${ }^{51}$ According to this model, in individuals having a biological and genetical vulnerability for psychiatric disorders, additional environmental stressors can exacerbate this vulnerability in the form of oversensitivity to stress, facilitating the manifestation of a psychiatric disorder. In this regard, history of CTEs was considered as possible severe environmental stressor having impacts on spesific brain regions such as the hypothalamic-pituitary-adrenal (HPA) axis, a system which is known to activate as a part of the stress response in individuals. ${ }^{52}$ History of CTEs can result in impaired HPA axis stress response in adulthood. Thus, HPA axis provides possible mechanisms through which CTEs could aggravate the underlying biological risk factors implicit in schizophrenia, putting individuals at higher risk for experiencing psychotic symptoms. Although the neural underpinnings of auditory hallucinations are still mostly unclear, some researchers have speculated that the networks involved in auditory hallucinations are particularly affected by the stress of CTEs, resulting in an even higher risk for the experience of auditory hallucinations. ${ }^{20}$ The other important model that can explain the associations between traumatic life events such as CTEs and schizophrenia is traumatagenic neurodevelopmental (TN) model presenting the similarities between the effects of traumatic events on the developing brain and the biological abnormalities in schizophrenia patients, such as overactivity of the HPA axis; abnormalities of neurotransmitters and brain structures. ${ }^{14,15}$

There was a significant positive correlation between persecutory delusions and CTQ-total and all subscales of CTQ28. Also, there was a significant positive correlation between delusions of reference and CTQ-total, and all subscales of CTQ-28. However somatic delusions were moderately positivitely correlated only with CSA consistent with the literature. ${ }^{9,48-50,53}$ In the analysis of the UK 2007 Adult Psychiatric Morbidity Survey, it was suggested that paranoia was specifically associated with CPA. ${ }^{49}$ Bentall and Fernyhough ${ }^{54}$ note that paranoia is linked to heightened threat over-anticipation and a tendency to attribute negative experiences to external sources. Recently in a review conducted by Bentall et al., ${ }^{48}$ attachment styles thought to have specific effects on the development of paranoid symptoms. One possible explanation for the association between attachment and paranoid thoughts (delusion of persecution), the attachment might be expected to have effects on the developing self-concept and the ability to trust others. ${ }^{48}$ Literature suggests the evidence that paranoid beliefs arise in the context of insecure attachment. ${ }^{49}$ Consistent with this, in a non-clinical sample researchers have revealed the differential associations between paranoid beliefs and neglect and that insecure attachment mediates the relationship between neglect and paranoia. ${ }^{50}$ Recently, in a study conducted by Wicham et al., ${ }^{53}$ the insecure attachment was found to be particularly associated with paranoid delusions. Examing the relevant literature, we have noticed that association between paranoid delusions and CTEs particularly CPA and neglect was thought to be mediated through the insecure attachment styles. ${ }^{48-50}$ It is important to take into account the attachment styles of the schziophrenic patients particulary admitting with paranoid delusions while making the treatment plan.

There was a moderately significant positive correlation between CTQ total scores and ability to feel intimacy and closeness; relationship with friends and peers consistent with the findings about negative symptoms and CTEs. ${ }^{44}$

In the patient group, there was a significant negative correlation between CTQ total scores and GAF and CGI-S consistent with the other studies. ${ }^{23,43,44}$ Studies have consistently suggested that history of CTEs has an important effect on global functioning ${ }^{23,43}$ and may lead poorer patient outcomes. ${ }^{43}$

There was a moderately significant positive correlation between CTQ-28 total and CTQ-28 subscales and DES total scores. In a study conducted by Schafer et al..$^{55}$ for evaluating the correlations between CTEs and dissociation in patients with schizophrenia spectrum disorders, researchers have suggested that CPN and CEA showed significant correlations with dissociative symptoms at admission. When patients were stabilized, about a month, CEA still showed a significant correlation with dissociative symptoms. ${ }^{10,56}$

Dissociation and psychosis can be a different diagnosis, or they can be synonymous structures or overlapping structures with common and different properties. Schneiderian symptoms of schizophrenia (auditory hallucinations, thought withdrawal, thought insertion, thought broadcasting, etc.) are also common in dissociative disorders. Dissociation and psychosis are not always but usually, occur as a result of traumatic experiences. ${ }^{55}$ Dissociative experiences were significantly higher in the patient group, consistent with the literature. ${ }^{10,25-28}$ This may be related to the high rate of CTEs in the patient group and its causal relationship with dissociation. There was a moderately 
significant positive correlation between some negative symptoms (e.g., ability to feel intimacy and closeness, relationship with friends and peers) and both CTQ total scores and DES scores. Since the dissociative symptoms have similarities and differences with the psychotic symptoms, this may have resulted in higher DES scores in the patient group. ${ }^{55}$

Finally, we tested if there is relationship between dissociation, CTEs and psychotic symptoms. There was a moderately significant positive correlation between DES total score and delusions (persecutory delusions, delusions of reference), consistent with the literature. ${ }^{56}$ In contrast with some studies, we did not find any significant association between dissociative experiences and hallucinations. ${ }^{57-59}$ In a sudy conducted by Perona-Garcelán et al. ${ }^{59} \mathrm{CTEs}$ was found to be positively correlated with the dissociation, hallucination, and delusions. Moreover, it was indicated that the dissociation was suggested to be a potential mediator between CTEs and hallucinations. ${ }^{59}$ Also regarding auditory hallucinations, researchers have suggested that spesific adversity, particularly CSA, may inhibit the source-monitoring mechanisms required to differentiate between external and self-generated stimuli, possibly in combination with adversity-induced dissociation. ${ }^{48}$

The TN model suggests potential explanations for other findings in schizophrenia research beyond oversensitivity to stress, including cognitive impairment, pathways to positive and negative symptoms, and the association between psychotic symptoms and dissociation. ${ }^{14}$ Negative life events almost certainly do not have "pure" psychological effects. Negative life events tend to co-occur so that, for example, a child who is sexually abused is at increased risk of being bullied at school. Also, symptoms can co-occur because the experience of one symptom can facilitate the occurrence of another. ${ }^{55}$ It is necessary to analyze further the causal link between CTEs and Schneiderian symptoms and dissociative symptoms in a large population-based prospective study.

\section{Limitations}

There are some limitations to our study. Our sample represents a regularly monitored group in tertiary health care, so it can not be generalized to the whole sample of schizophrenia patients. So, the findings could not be generalized to all patients. Another limitation of this study is the small sample size. Bonferroni correction is calculated as p-value/number of comparisons and made to reduce the type 1 error. ${ }^{60}$ Bonferroni correction has not been made in comparisons in our study. There are, however, some debates in this regard, as it can increase type II error, cause significant differences to be considered insignificant, reduce statistical power, and cause bias. Therefore, publications are suggesting that Bonferroni correction should not be done. ${ }^{61}$ Then, we preferred not to do Bonferroni correc- tion in our study.

\section{Conclusion}

Our findings imply that exposure to CTEs particularly abuse should be regarded as an essential determinant of schizophrenia. Our results suggest that clinicians should routinely inquire about CTEs in order to develop comprehensive formulations and treatment plans when working with patients with schizophrenia or similar diagnoses. Psychosocial interventions which have been used for patients affected by trauma might be considered among the treatment options for patients with psychosis. Clinicians should keep in mind CTEs as a crucial environmental stressor in the etiologic and pathophysiologic process of schizophrenia. This is particularly important for making comprehensive formulations and appropriate treatment strategy (e.g., trauma-focused interventions) in psychotic patients.

\section{Conflicts of Interest}

The authors have no potential conflicts of interest to disclose.

\section{Author Contributions}

Zeynep Yildiz Akbey: Collecting data, statistical analysis, finding the references. Mustafa Yildiz: Designing and mentoring the study, revising the manuscript. Nermin Gündüz:Writing the manuscript.

\section{ORCID iDs}

Nermin Gündüz

Zeynep Yildiz Akbey

https://orcid.org/0000-0002-0188-6232

Mustafa Yildiz

https://orcid.org/0000-0002-3476-1487

https://orcid.org/0000-0003-0769-1628

\section{REFERENCES}

1. Wigman JT, van Winkel R, Jacobs N, Wichers M, Derom C, Thiery E, et al. A twin study of genetic and environmental determinants of abnormal persistence of psychotic experiences in young adulthood. Am J Med Genet B Neuropsychiatr Genet 2011;156B:546-552.

2. Bendall S, Jackson HJ, Hulbert CA, McGorry PD. Childhood trauma and psychotic disorders: a systematic, critical review of the evidence. Schizophr Bull 2008;34:568-579.

3. Longden E, Sampson M, Read J. Childhood adversity and psychosis: generalised or specific effects? Epidemiol Psychiatr Sci 2016;25:349-359.

4. Shevlin M, Houston JE, Dorahy MJ, Adamson G. Cumulative traumas and psychosis: an analysis of the national comorbidity survey and the British Psychiatric Morbidity Survey. Schizophr Bull 2008; 34:193-199.

5. Varese F, Smeets F, Drukker M, Lieverse R, Lataster T, Viechtbauer W, et al. Childhood trauma increases the risk of psychosis: a meta-analysis of patient-control, prospective- and cross sectional cohort studies Schizophr Bull 2012;38:661-671.

6. Matheson SL, Shepherd AM, Pinchbeck RM, Laurens KR, Carr VJ. Childhood adversity in schizophrenia: a systematic meta-analysis Psychol Med 2013;43:225-238.

7. Bonoldi I, Simeone E, Rocchetti M, Codjoe L, Rossi G, Gambi F, et al. Prevalence of self-reported childhood abuse in psychosis: a meta-analysis of retrospective studies. Psychiatry Res 2013;210:8-15.

8. Duhig M, Patterson S, Connell M, Foley S, Capra C, Dark F, et al. The prevalence and correlates of childhood trauma in patients with early psychosis. Aust N Z J Psychiatry 2015;49:651-659.

9. Ucok A, Bikmaz S. The effects of childhood trauma in patients with first 
episode schizophrenia. Acta Psychiatr Scand 2007;116:371-377.

10. Sar V, Taycan O, Bolat N, Ozmen M, Duran A, Oztürk E, et al. Childhood trauma and dissociation in schizophrenia. Psychopathology 2010; 43:33-40.

11. Arseneault L, Cannon M, Fisher HL, Polanczyk G, Moffitt TE, Caspi A. Childhood trauma and children's emerging psychotic symptoms: A genetically sensitive longitudinal cohort study. Am J Psychiatry 2011;168: 65-72.

12. Janssen I, Krabbendam L, Bak M, Hanssen M, Vollebergh W, de Graaf $\mathrm{R}$, et al. Childhood abuse as a risk factor for psychotic experiences. Acta Psychiatr Scand 2004;109:38-45.

13. Cutajar MC, Mullen PE, Ogloff JR, Thomas SD, Wells DL, Spataro J. Schizophrenia and other psychotic disorders in a cohort of sexually abused children. Arch Gen Psychiatry 2010;67:1114-1119.

14. Read J, Perry BD, Moskowitz A, Connolly J. A traumagenic neurodevelopmental model of schizophrenia. Psychiatry Interpers Biol Process 2001; 64:319-345.

15. Read J, Fosse R, Moskowitz A, Perry B. The traumagenic neurodevelopmental model of psychosis revisited. Neuropsychiatry 2014;4:65-79.

16. Gallagher BJ, Jones BJ. Childhood stressors and symptoms of schizophrenia. Clin Schizophr Relat Psychoses 2013;7:124-130.

17. Wang Z, Xue Z, Pu W, Yang B, Li L, Yi W, et al.Comparison of first episode and chronic patients diagnosed with schizophrenia: Symptoms and childhood trauma. Early Interv Psychiatry 2013;7:23-30.

18. Burns JK, Jhazbhay K, Esterhuizen T, Emsley R. Exposure to trauma and the clinical presentation of first-episode psychosis in South Africa. J Psychiatr Res 2011;45:179-184.

19. Daalman K, van Zandvoort M, Bootsman F, Boks M, Kahn R, Sommer I. Auditory verbal hallucinations and cognitive functioning in healthy individuals. Schizophr Res 2011;132: 203-207.

20. Sheffield JM, Williams LE, Blackford JU, Heckers S. Childhood sexual abuse increases the risk of auditory hallucinations in psychotic disorders. Compr Psychiatry 2013;54:1098-1104.

21. Read J, Agar K, Argyle N, Aderhold V. Sexual and physical abuse during childhood and adulthood as predictors of hallucinations, delusions and thought disorder. Psychol Psychother 2003;76:1-22.

22. Schenkel LS, Spaulding WD, DiLillo D, Silverstein SM. Histories of childhood maltreatment in schizophrenia: Relationships with premorbid functioning, symptomatology, and cognitive deficits. Schizophr Res 2005; 76:273-286.

23. Van Nierop M, Viechtbauer W, Gunther N, von Zelst C, de Graaf R, Ten Have M, et al. Childhood trauma is associated with a specific admixture of affective, anxiety, and psychosis symptoms cutting across traditional diagnostic boundaries. Psychol Med 2015;45:1277-1288.

24. Sideli L, Mule A, La Barbera D, Murray RM. Do child abuse and maltreatment increase risk of schizophrenia? Psychiatry Investig 2012;9: 87-99.

25. Vogel M, Schatz D, Spitzer C, Kuwert P, Moller B, Freyberger HJ, et al. A more proximal impact of dissociation than of trauma and posttraumatic stress disorder on schneiderian symptoms in patients diagnosed with schizophrenia. Compr Psychiatry 2009;50:128-134.

26. Cole CL, Newman TK, Kennedy F. Dissociation mediates the relationship between childhood maltreatment and subclinical psychosis J Trauma Dissociation 2016;17:577-592.

27. Schafer I, Fisher HL, Aderhold V, Huber B, Hoffmann-Langer L, Golks $\mathrm{D}$, et al. Dissociative symptoms in patients with schizophrenia: Relationships with childhood trauma and psychotic symptoms. Compr Psychiatry 2012;53:364-371.

28. Sahin S, Yuksel C, Guler J, Karadayi G, Akturan G, Gode E, et al. The history of childhood trauma among individuals with ultra high risk for psychosis is as common as among patients with first-episode schizophrenia. Early Interv Psychiatry 2013;7:414-420.

29. Rajkumar RP. The impact of childhood adversity on the clinical features of schizophrenia. Schizophr Res Treatment 2015;2015:532082.

30. American Psychiatric Association. Diagnostic and Statistical Manual of
Mental Disorders. Fourth Edition, Text Revision (DSM-IV-TR). Washington DC: American Psychiatric Association; 2000.

31. Bernstein EM, Putnam PW. Development, reliability, and validity of a dissociation scale. J Nerv Ment Dis 1986;174:727-735.

32. Yargıç LI, Tutkun H, Sar V. The reliability and validity of the Turkish version of the dissociative experiences scale. Dissociation 1995;8:10-12.

33. Bernstein EM, Fink L, Handelsman L, Lovejoy M, Wenzel K, et al. Initial reliability and validity of a new retrospective measure of child abuse and neglect. Am J Psychiatry 1994;151:1132-1136.

34. Şar V, Öztürk E, İkikardeș E. Validity and reliability of the Turkish version of childhood trauma questionnaire. Türkiye Klinikleri J Med Sci 2012;32:1054-1063.

35. Andreasen NC. Methods for assessing positive and negative symptoms. Mod Probl Pharmacopsychiatry 1990;24:73-88.

36. Erkoç Ş, Arkonaç O, Ataklı C, Özmen E. The reliability and validity of scale for the assesment of the positive symtoms. Düşünen Adam 1991a;4:20-24.

37. Erkoç Ş, Arkonaç O, Atakl C, Özmen E. The reliability and validity of scale for the assesment of the negative symstoms. Düşünen Adam 1991b;4:16-18.

38. First MB, Spitzer RL, Gibbon M, Williams JBW. Structured Clinical Interview for DSM-IV Axis 1 Disorders, Clinical Version (SCID-CV). Washington: American Psychiatric Press; 1996.

39. Özkürkçügil A, Aydemir Ö, Yıldız M, Esen Danacı A, Köroğlu E. DSMIV Eksen I Bozuklukları İçin Yapılandırılmış Klinik Görüşmenin Türkçe’ ye Uyarlanması Ve Güvenilirlik Çalışması. İlaç Ve Tedavi Dergisi 1999;12:233-236.

40. Guy W. The Clinical Global Impression Scale. ECDEU Assessment Manual for Psychopharmacology. Rockville, MD: US Department of Heath, Education, and Welfare Public Health Service Alcohol, Drug Abuse, and Mental Health Administration, NIMH Publication, 1976, p.218-222.

41. Asuero AG, Sayago A, Gonzalez AG. The correlation coefficient: an overview. Crit Rev Anal Chem 2006;36:41-59.

42. Fisher H, Morgan C, Dazzan P, Craig T, Morgan K, Hutchinson G, et al. Gender differences in the association between childhood abuse and psychosis. Br J Psychiatry 2009;194:319-325.

43. Trauelsen AM, Bendall S, Jansen JE, Nielsen HG, Pedersen MB, Trier $\mathrm{CH}$, et al. Childhood adversities: Social support, premorbid functioning and social outcome in first-episode psychosis and a matched casecontrol group. Aust N Z J Psychiatry 2016;50:770-782.

44. Alameda L, Ferrari C, Baumann PS, Gholam-Rezaee M, Do KQ, Conus P. Childhood sexual and physical abuse: Age at exposure modulates impact on functional outcome in early psychosis patients. Psychol Med 2015;45:2727-2736.

45. Viola TW, Salum GA, Kluwe-Schiavona B, Sanvicente-Vieiraa B, Levandowski ML, Grassi-Oliveira R. The influence of geographical and economic factors in estimates of childhood abuse and neglect using the childhood trauma questionnaire: a worldwide meta-regression analysis. Child Abuse Negl 2016; 51:1-16.

46. Hardt J, Rutter M. Validity of adult retrospective reports of adverse childhood experiences: a review of the evidence. J Child Psychol Psychiatry 2004;45:260-273.

47. Fisher HL, Craig TK, Fearon P, Morgan K, Dazzan P, Lappin J, et al. Reliability and comparability of psychosis patients' retrospective reports of childhood abuse. Schizophr Bull 2011;37:546-553.

48. Bentall RP, de Sousa P, Varese F, Wickham S, Sitko K, Haarmans M, et al. From adversity to psychosis: pathways and mechanisms from specific adversities to specific symptoms. Soc Psychiatry Psychiatr Epidemiol 2014;49:1011-1022.

49. Bentall RP, Wickham S, Shevlin M, Varese F. Do specific early-life adversities lead to specific symptoms of psychosis? A study from the 2007 the Adult Psychiatric Morbidity Survey. Schizophr Bull 2012;38:734-740.

50. Sitko K, Bentall RP, Shevlin M, Sellwood W. Associations between specific psychotic symptoms and specific childhood adversities are mediated by attachment styles: an analysis of the National Comorbidity Survey. Psychiatr Res 2014;217:202-209. 
51. Mirsky AF, Duncan CC. Etiology and expression of schizophrenia: neurobiological and psychosocial factors. Annu Rev Psychol 1986;37:291319.

52. Charmandari E, Kino T, Souvatzoglou E, Chrousos GP. Pediatric stress: hormonal mediators and human development. Horm Res 2003;59:161179.

53. Wickham S, Sitko K, Bentall RP. Insecure attachment is associated with paranoia but not hallucinations in psychotic patients: the mediating role of negative self esteem. Psychol Med 2015;45:1495-1507.

54. Bentall RP, Fernyhough C. Social predictors of psychotic experiences: specificity and psychological mechanisms. Schizophr Bull 2008;34:10091011.

55. Schafer I, Harfst T, Aderhold V, Briken P, Lehmann M, Moritz S, et al. Childhood trauma and dissociation in female patients with schizophrenia spectrum disorders an exploratory study. J Nerv Ment Dis 2006;194: 135-138.

56. Vogel M, Spitzer C, Kuwert P, Möller B, Freyberger HJ, Grabe HJ. Asso- ciation of childhood neglect with adult dissociation in schizophrenic inpatients. Psychopathology 2009;42:124-130.

57. Varese F, Udachina A, Myin-Germeys I, Oorschot M, Bentall RP. The relationship between dissociation and auditory verbal hallucinations in the flow of daily life in patients with psychosis. Psychosis 2011;3:14-28.

58. Varese F, Barkus E, Bentall RP. Dissociation mediates the relationship between childhood trauma and hallucination proneness. Psychol Med 2012;42:1025-1036.

59. Perona-Garcelán S, Carrascoso-Lopez F, Garcia-Montes JM, DuctorRecuerda MJ, Jimenez AML, Vallina-Fernandez O, et al. Dissociative experiences as mediators between childhood trauma and auditory hallucinations. J Trauma Stress 2012;25:323-329.

60. Aickin M, Gensler H. Adjusting for multiple testing when reporting research results: the Bonferroni vs. Holm methods. Am J Public Health 1996;86:726-728.

61. Nakagawa SA. Farewell to Bonferroni: the problems of low statistical power and publication bias. Behav Ecol 2004;15:1044-1045. 